University of Montana

ScholarWorks at University of Montana

Chemistry and Biochemistry Faculty

Publications

Chemistry and Biochemistry

$5-15-2002$

\title{
Magnetization Reversal of Elliptical Co/Cu/Co Pseudo-Spin Valve Dots
}

NgocNga Dao

University of New Orleans

Scott L. Whittenburg

University of Montana - Missoula, scott.whittenburg@umontana.edu

Y. Hao

Massachusetts Institute of Technology

Leszek M. Malkinski

University of Colorado at Colorado Springs

Jian Qing Wang

University of New Orleans

See next page for additional authors

Follow this and additional works at: https://scholarworks.umt.edu/chem_pubs

Part of the Biochemistry Commons, Chemistry Commons, and the Physics Commons Let us know how access to this document benefits you.

\section{Recommended Citation}

Dao, NgocNga; Whittenburg, Scott L.; Hao, Y.; Malkinski, Leszek M.; Wang, Jian Qing; and Ross, C. A., "Magnetization Reversal of Elliptical Co/Cu/Co Pseudo-Spin Valve Dots" (2002). Chemistry and Biochemistry Faculty Publications. 66.

https://scholarworks.umt.edu/chem_pubs/66

This Article is brought to you for free and open access by the Chemistry and Biochemistry at ScholarWorks at University of Montana. It has been accepted for inclusion in Chemistry and Biochemistry Faculty Publications by an authorized administrator of ScholarWorks at University of Montana. For more information, please contact scholarworks@mso.umt.edu. 
Authors

NgocNga Dao, Scott L. Whittenburg, Y. Hao, Leszek M. Malkinski, Jian Qing Wang, and C. A. Ross

This article is available at ScholarWorks at University of Montana: https://scholarworks.umt.edu/chem_pubs/66 


\title{
Magnetization reversal of elliptical $\mathrm{Co} / \mathrm{Cu} / \mathrm{Co}$ pseudo-spin valve dots
}

\author{
N. Dao and S. L. Whittenburga \\ Department of Chemistry/AMRI, University of New Orleans, New Orleans, Louisiana 70148 \\ Y. Hao and C. A. Ross \\ Department of Materials Science and Engineering, Massachusetts Institute of Technology, Cambridge, \\ Massachusetts 02139 \\ L. M. Malkinski \\ Department of Physics, University of Colorado, Colorado Springs, Colorado 80933 \\ J. Q. Wang \\ Advanced Materials Research Institute, University of New Orleans, New Orleans, Louisiana 70148
}

\begin{abstract}
We present our recent simulated results on $\mathrm{Cr}(5 \mathrm{~nm}) / \mathrm{Cu}(5 \mathrm{~nm}) / \mathrm{Co}(5 \mathrm{~nm}) / \mathrm{Cu}(3 \mathrm{~nm}) / \mathrm{Co}(2 \mathrm{~nm})$ pseudo-spin valve dots. The simulated results agree qualitatively with the experimental results. Three different sizes of elliptical dots, $150 \mathrm{~nm} \times 105 \mathrm{~nm}, 175 \mathrm{~nm} \times 70 \mathrm{~nm}$, and $200 \mathrm{~nm} \times 60 \mathrm{~nm}$, were simulated. Our simulations show that in these types of dots magnetization reversal occurs by the formation of domain walls: $90^{\circ}$ for $175 \mathrm{~nm} \times 70 \mathrm{~nm}$ and $360^{\circ}$ for $200 \mathrm{~nm} \times 60 \mathrm{~nm}$. No domain wall was observed in the reversal of the $150 \mathrm{~nm} \times 105 \mathrm{~nm}$ dots. For such dots, the simulated loops show a small two-step reversal pattern with the thin upper layer partially reversing followed by complete reversal of both layers at higher fields. In the larger dots, a two-step reversal is clearly observed both in the simulation and experiment. (c) 2002 American Institute of Physics.
\end{abstract}

[DOI: $10.1063 / 1.1454984]$

\section{INTRODUCTION}

A magnetic spin-valve structure consists of two ferromagnetic layers separated by a nonmagnetic layer. ${ }^{1}$ Spin valves have numerous technological applications including transistors ${ }^{2}$ and read heads for computer disk drives. ${ }^{3}$ A spin valve without the antiferromagnetic layer or pseudo-spin valve, which is nondestructive to the readout, has been applied to magneto-resistive random access memory. ${ }^{4} \mathrm{Re}-$ cently, pseudo-spin valve material has served as the working layer for patterned media with potential application in ultrahigh density storage. Before such media can be used in such applications, however, several issues need to be addressed including thermal stability and the effect of the shape of the magnetic element on magnetic domain structure and the switching mechanism. In this study the switching mechanism and the underlying magnetic domain structure of elliptical dots of pseudo-spin valve material with three different aspect ratios were studied using micromagnetics simulation. Such simulations can yield valuable information into the magnetization reversal mechanism in these complicated structures.

\section{PROCEDURE}

Recent experimental work has been presented on patterned elliptical dots of $\mathrm{Cr}(5 \mathrm{~nm}) / \mathrm{Cu}(5 \mathrm{~nm}) / \mathrm{Co}$ (bottom, 5 $\mathrm{nm}) / \mathrm{Cu}(3 \mathrm{~nm}) / \mathrm{Co}$ (top, $2 \mathrm{~nm}$ ) pseudo-spin valve material created using interference lithography. ${ }^{5}$ A vibrating sample magnetometer was used to obtain the experimental hysteresis loops. The aspect ratio of the simulated dots matches those of the experimental dots, $105 \mathrm{~nm} \times 150 \mathrm{~nm}, \quad 175 \mathrm{~nm}$ $\times 70 \mathrm{~nm}$ and $200 \mathrm{~nm} \times 60 \mathrm{~nm}$. All calculations were per-

${ }^{a)}$ Electronic mail: swhitten@uno.edu formed using the three-dimensional micromagnetics code OOMMF (the object-oriented micromagnetic framework) obtained from the National Institute of Standards and Technology. ${ }^{6}$ The input file for the simulations was generated using Java micromagnetics. ${ }^{7}$ OOMMF discretizes the sample into a uniform rectangular mesh. In these simulations the discretization cell is $5 \mathrm{~nm} \times 5 \mathrm{~nm} \times 1 \mathrm{~nm}$ in the $x, y$, and $z$ directions. The $z$ direction is taken as the direction perpendicular to the pseudo-spin-valve layers. The $x$ direction is the long axis of the ellipse. Although the cell size used in the simulation is much less than the exchange length for cobalt, a discretization of $1 \mathrm{~nm}$ in the $z$ direction means that only two layers of cells are used to simulate the upper layer. As a test of this choice of discretization, a cell size of $5 \mathrm{~nm}$ $\times 5 \mathrm{~nm} \times 0.5 \mathrm{~nm}$ was employed for a simulation of a $150 \mathrm{~nm} \times 105 \mathrm{~nm}$ dot. The results were identical to those obtained using the larger cell size and suggest that discretization errors are minimal.

The OOMMF code allows the magnetization of the elliptical dot to be calculated as a function of time by numerical solution of the Landau-Lifshitz-Gilbert (LLG) equation using an Euler-type solver. The magnetization in each cell is determined by the local magnetic field in the cell. The local field has contributions from the anisotropy, exchange, magnetostatic and applied fields. The anisotropy field is correct to first order and the default value of $K_{1}=5.2 \times 10^{5} \mathrm{~J} / \mathrm{m}^{3}$ of cobalt was chosen for study. The exchange field is computed using the six nearest neighbors and the default exchange coupling constant of $A=3.0 \times 10^{-11} \mathrm{~J} / \mathrm{m}$ was used. The default value of the saturation magnetization, $M_{s}=1.4 \times 10^{6} \mathrm{~A} / \mathrm{m}$ was also used. Since the exchange field only includes nearest neighbors it cannot account for the coupling between the layers. Long-range interactions are included in the magneto- 


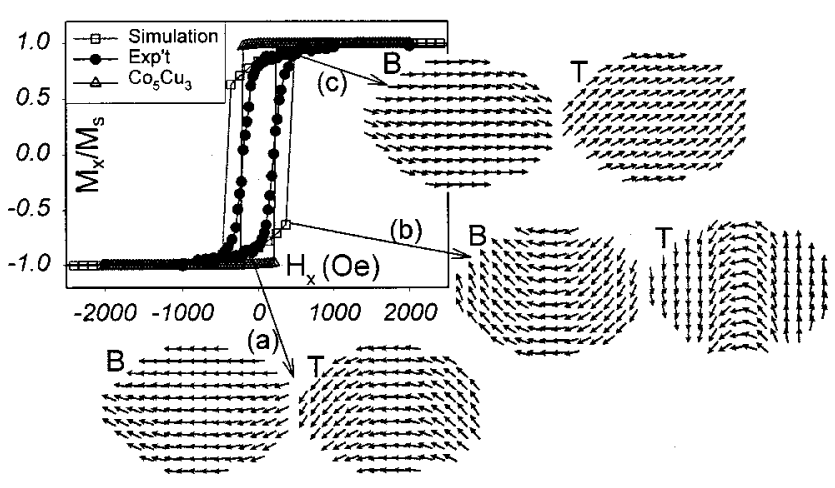

FIG. 1. The simulated loop (hollow) and the experimental loop (solid) for the $150 \mathrm{~nm} \times 105 \mathrm{~nm}$ dot. Magnetic domains at three states are presented. B for the bottom layer, and $\mathrm{T}$ for the top layer. (a) Before the remanent state. (b) The top layer begins to annihilate $\mathrm{C}$ state. (c) Just after reversal. Every second spin is shown. The simulated loop without the top layer of $\mathrm{Co}_{5} \mathrm{Cu}_{3}$ (triangle, hollow symbol) is also shown for discussion in the text.

static or demagnetizing field. OOMMF uses a method due to Newell to calculate the magnetostatic interaction. ${ }^{8}$ The major hysteresis loop was calculated by following the equilibrium states of the magnetization obtained from the solution of the LLG equation as the applied field was swept from the saturated value of 300 to $-300 \mathrm{mT}$ and back and plotting the magnetization at each field point versus the applied field.

\section{RESULTS AND DISCUSSION}

Hysteresis loops along the long axis and the short axis of the elliptical dots were simulated, however, the loops corresponding to field along the short axis displayed little coercivity and correspond to free rotation of the magnetization. Here we discuss only the simulations where the applied field is along the long axis of the elliptical dots, $H_{x}$.

The hysteresis loop for the lowest aspect ratio dot, the $105 \mathrm{~nm} \times 150 \mathrm{~nm}$ dot, is shown in Fig. 1. Also shown in this figure are several magnetic domains that occur in the top and bottom layers. In the experimental loop, there is no evidence of a difference in the switching of the two levels. The simulation, however, suggests that the top layer passes through a $C$ magnetization state before both layers switch. There are two noticeable differences between the simulated and experimental loops; the coercivity of the simulated loops is too large and the experimental loops have a larger "tilt." Two possible explanations for the difference in the coercivity are the choice of bulk cobalt anisotropy parameters (including the neglect of the second order anisotropy constant and temperature dependence of the anisotropy constants in the simulation) and the fact that the top layer in the experimental sample may be oxidized. This sample was not capped and, therefore, the upper layer may be oxidized as discussed below in the larger aspect ratio dots. This can be checked by performing a simulation only on the lower layer. The result is shown in Fig. 1. The agreement with the experimental coercivity is significantly improved. The tilting of the experimental hysteresis loops for arrays of similar magnetic elements has been extensively studied. ${ }^{9}$ The tilt arises from long-range dipolar or magnetostatic interaction between the elements

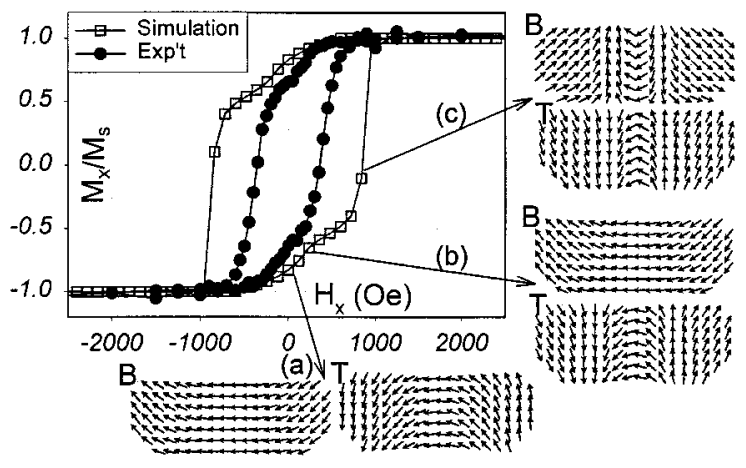

FIG. 2. Both the simulated (hollow) and experimental (solid) results are plotted for the $175 \mathrm{~nm} \times 70 \mathrm{~nm}$ dot. Three magnetic domains at three states are indicated where $\mathrm{B}$ is for the bottom layer, $\mathrm{T}$ is for the top layer. Every second spin is shown. (a) At the remanent state. (b) At the first switch. (c) At the second switch.

and from variability between elements. While the OOMMF code does not include periodic boundary conditions making more realistic simulation of the experimental dot arrays possible, we have previously demonstrated that by including a greater number of dots in the simulation we can demonstrate that the long-range magnetostatic interaction can produce a tilt in the simulated loops. ${ }^{10}$

The occurrence of the $C$ state is also observed in the simulation of the magnetization reversal of the $70 \mathrm{~nm}$ $\times 175 \mathrm{~nm}$ pseudo-spin valve dot. The experimental hysteresis loop and the simulated loop are shown in Fig. 2. Also shown are three magnetization domains occurring in the top and bottom layers during the reversal process. The initial decrease in the magnetization occurring during the reversal near zero applied field corresponds to formation of a $C$ state in both the top and bottom layers. Because these two layers are antiferromagnetically coupled, the magnetization direction of the spins in the layers has an opposite sense. This opposite sense coupling of the soft and hard layers leading to a $C$ state (this is "up" in one layer and "down" in the other layer) in sandwich-type structures has been observed in other very recent simulations. ${ }^{11}$ What appears as the switching of the top layer in the experimental loop is actually the formation of a $90^{\circ}$ domain wall in the top layer. Note that the applied field at this point is not sufficient to cause complete switching in either layer. The zero magnetization state occurs via formation of a $90^{\circ}$ wall in the bottom layer. Again, because of the antiferromagnetic coupling between the layers, the vector sense of the two layers is opposite, leading to cancellation of the magnetization vectors. Eventually the applied field is sufficient to reverse both layers and the large drop in the magnetization occurs as the domain walls in both layers are annihilated.

The experimental and simulated hysteresis loops for the highest aspect ratio dots, $60 \mathrm{~nm} \times 200 \mathrm{~nm}$, are shown in Fig. 3. Unfortunately the top layer in the experimental sample is most likely oxidized so direct comparison between the experimental and simulated loops is not possible. However, we can use the simulation to predict what should be observed. The magnetization reversal process in this sample is very similar to the $70 \mathrm{~nm} \times 175 \mathrm{~nm}$ dot. A $C$ state is observed in 


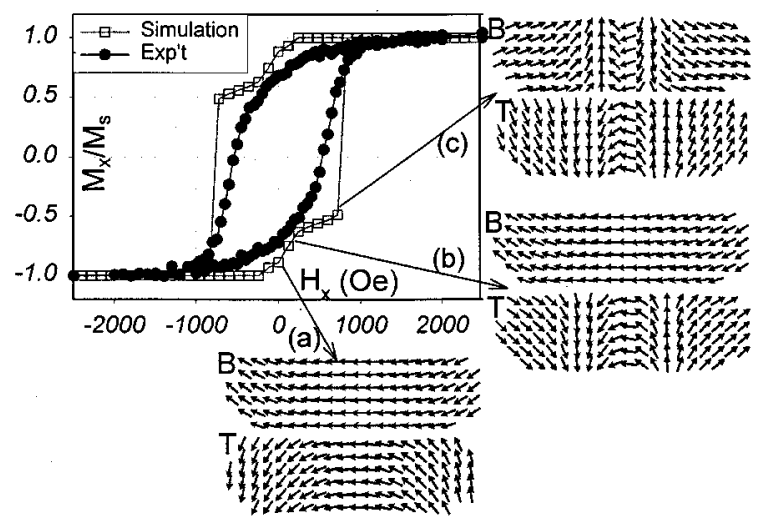

FIG. 3. Hysteresis loops of the simulation (hollow) and experiment (solid) are shown for the $200 \mathrm{~nm} \times 60 \mathrm{~nm}$ dot. Magnetic domains for three states are also presented with B for the bottom layer and T for the top layer. (a) At the remanent state. (b) At the first switch where a $90^{\circ}$ domain wall appears on the top layer. (c) At the second switch, a $360^{\circ}$ domain wall occurs at the bottom layer with the annihilation of $90^{\circ}$ wall at the top layer. Every second spin is shown.

both layers with a more pronounced curvature in the top layer. This is followed by the formation of a $90^{\circ}$ wall in the top layer. In the high aspect ratio dots this is followed by the formation of a $360^{\circ}$ wall in the bottom layer. Because the spins are in the plane of the film, this is a Néel wall. The $360^{\circ}$ wall is believed to be nucleated by inclusions ${ }^{12}$ and has been observed in previous modeling of $\mathrm{Co} / \mathrm{Cu}$ multilayers including defects in the film structure. ${ }^{13}$ The domain walls in the spin-valve structures are parallel to the magnetocrystalline anisotropy direction. This is in agreement with the results from earlier experimental studies on $\mathrm{Ni}_{80} \mathrm{Fe}_{20} / \mathrm{Cu} / \mathrm{Co} .{ }^{14}$ As the applied field is increased, the domain walls in both layers annihilate and the dot saturates.

Several important factors have not been included in this study. Unfortunately, the full coupling between the two layers is probably not fully accounted for in our simulation due to the modeling of the exchange interaction via a nearestneighbor interaction. Although small, long-range exchange coupling should be considered. The magnitude of the effect can be calculated using canonical tight-binding bands. ${ }^{15}$ For the $3 \mathrm{~nm} \mathrm{Cu}$ thickness in these samples we can estimate the long-range exchange coupling constant to be on the order of $1.5 \times 10^{-13} \mathrm{~J} / \mathrm{m}$. Thus, the long-range coupling is quite small. Why then do the two layers antiferromagnetically couple in our simulations? The antiparallel orientation is due purely to the magnetostatic coupling between the two layers. Finally, interface anisotropy is not included in our simulations. The magneto-crystalline anisotropy is the major contribution to the calculated anisotropy energy.

\section{CONCLUSIONS}

Despite the shortcomings in the model, the agreement between the experimental and simulated loops is quite good. Quantitative agreement could be obtained if the anisotropy constant were allowed to deviate from the default, bulk cobalt value chosen for this study. In a qualitative sense the magnetization reversal process can be understood from these simulations. For low aspect ratio dots the switching in both layers occurs almost simultaneously. A $C$ magnetization state occurs in the thin layer just prior to switching, however, this state is difficult to observe experimentally due to the small change in the magnetization, which is probably obscured by the different switching fields in an array of such elliptical dots. For higher aspect ratio dots a clear multi-step process is observed in the magnetization reversal process. A $C$ state occurs in both layers followed by formation of a domain wall in the top layer. The zero magnetization state in the $175 \mathrm{~nm}$ $\times 70 \mathrm{~nm}$ dot then corresponds to formation of a domain wall in the bottom layer. As the applied field is increased the domain walls in both layers are annihilated, leading to reversal and saturation.

\section{ACKNOWLEDGMENT}

The authors acknowledge the support of this work by AMRI through DoD/DARPA Grant No. MDA972-97-0003.

${ }^{1}$ B. Dieny, V. S. Speriosu, S. S. P. Parkin, B. A. Gurney, D. R. Wilhoit, and D. Mauri, Phys. Rev. B 43, 1297 (1991).

${ }^{2}$ R. Jansen, O. M. J. van't Rrve, S. D. Kim, R. Vlutters, P. S. Anil Kumar, and J. C. Lodder, J. Appl. Phys. 89, 7431 (2001).

${ }^{3}$ www.research.ibm.com/journals/rd42-1.html

${ }^{4}$ R. K. Romney, DARPA/UNO AMRI Review 2001 (New Orleans, LA: 02/21-02/23).

${ }^{5}$ Y. Hao, M. Walsh, M. Farhoud, C. A. Ross, H. I. Smith, J. Q. Wang, and L. Malkinski, IEEE Trans. Magn. 36, 2996 (2000).

${ }^{6} \mathrm{http}: / /$ math.nist.gov/oommf/

${ }^{7}$ www.JaMM.uno.edu

${ }^{8}$ A. J. Newell, W. Williams, and D. J. Dunlop, J. Geophys. Res., 98, 9551 (1993).

${ }^{9}$ M. Hwang, M. C. Abraham, T. A. Savas, H. I. Smith, R. J. Ram, and C. A. Ross, J. Appl. Phys. 87, 5108 (2000); M. Hwang, M. Farhoud, Y. Hao, M. Walsh, T. A. Savas, H. I. Smith, and C. A. Ross, IEEE Trans. Magn. 36, 3171 (2000).

${ }^{10}$ S. L. Whittenburg, N. Dao, and C. A. Ross, Physica B 306, 44 (2001).

${ }^{11}$ T.-N. Fang and J.-G. Zhu, J. Appl. Phys. 87, 7061 (2000); K. A. Zvezdin, J. Solid State 42, 120 (2000).

${ }^{12}$ D. O. Smith and K. J. Harte, J. Appl. Phys. 33, 1399 (1962).

${ }^{13}$ D. V. Berkov, N. L. Gorn, R. Mattheis, and T. Zimmermann, J. Magn. Magn. Mater. 182, 81 (1998).

${ }^{14}$ R. Kergoat, M. Labrune, J. Miltat, T. Valet, and J. C. Jacquet, J. Magn. Magn. Mater. 121, 339 (1993).

${ }^{15}$ J. Mathon, M. A. Villeret, J. M. Mander, D. M. Edwards, and R. B. Muniz, Mater. Res. Soc. Symp. Proc. 313, 171 (1993). 